\title{
A novel phase-noise-suppressed and delay-time-tunable mode-locked erbium-doped fiber laser
}

\author{
Gong-Ru Lin*, Yinchieh Lai \\ Institute of Electro-Optical Engineering, National Chiao Tung University, 1001 Ta Hsueh Rd., Hsinchu 300, Taiwan, ROC
}

Received 07 November 2001; received in revised form 06 August 2002; accepted 26 August 2002

\begin{abstract}
A phase-noise-suppressed and delay-time-tunable actively mode-locked erbium-doped fiber ring laser is demonstrated by using voltage-controlled and frequency-discriminated phase shifter as an inherent timing module. By changing the voltage from -3.35 to $3.6 \mathrm{~V}$, the maximum tuning range of phase and delay-time of $500 \mathrm{MHz}$ optical pulse-train from the laser can be up to $340^{\circ}(\sim 1.9 \pi)$ and 1.9 ns (nearly 1 period), respectively. Optimized responsivity and tuning deviation are $0.27 \mathrm{ps} / \mathrm{mV}$ and $\leqslant 4 \%$. The single-sided-band phase the laser operated at feedback controlled mode is significantly reduced to $-100 \mathrm{dBc} / \mathrm{Hz}$ (measured at frequency of $10 \mathrm{kHz}$ offset from center), which is at least $10 \mathrm{~dB}$ smaller than that of the laser operated at free-running mode.

(C) 2002 Published by Elsevier Science B.V.
\end{abstract}

Keywords: Erbium-doped; Fiber laser; Mode-locking; Phase noise; Phase-shift; Delay-time; Frequency-discriminated

Harmonic mode-locking has been considered as one of the promising techniques to construct highrepetitive optical pulse laser sources with relatively short pulsewidth [1-3]. It has been demonstrated that the mode-locked erbium-doped fiber lasers (EDFLs) based on this technique can provide stable and nearly transform-limited picosecond optical pulses with very low timing jitter [2]. These active harmonic mode-locked EDFLs have found versatile applications in ultra-high bit-rate optical transmission, high-speed optical time division

\footnotetext{
${ }^{*}$ Corresponding author. Tel.: +886-3-5712121 ext. 56376; fax: +886-3-5716631 or +886-2-28281873.

E-mail address: grlin@cc.nctu.edu.tw (G.-R. Lin).
}

multiplexed (OTDM) [4], as well as electro-optical sampling (EOS) [5] at the wavelength range around $1.55 \mu \mathrm{m}$ [6-11]. The typical optical pulsewidth of these lasers operated at $10 \mathrm{GHz}$ is around ps [6] and subpicosecond pulses can be obtained by using a dual-modulator scheme [7] or by external pulse compression techniques [8,9]. On the other hand, for the applications in phased-array antenna, wireless/fiber OTDM communication and optoelectronic sampling systems, the capability of continuously tuning the phase of microwave signals or optical clock via optical or optoelectronic techniques has been the main goal of many extensive research studies. The photonic microwave techniques can greatly improve the performance of the mechanical- or electronic-type microwave 
phase shifters developed in the early stage, which may be bulky, incapable of phase-tuning, and with narrow bandwidth or frequency-dependent operation. Recently, several types of true-time-delay optoelectronic phase-shifters (OEPSs) have been demonstrated by using different techniques like a set of optical fibers with different lengths or modified waveguide structures [12-14], a directly modulated laser-diode [15], and a modified integrated optical modulator $[16,17]$. Some of the OEPSs have already supported broadband (frequency-independent) operation and wide phasetuning range. To facilitate the compatibility of mode-locked EDFLs for the aforementioned applications, we demonstrate an inherently delaytime-tunable picosecond EDFL source by incorporating a voltage-controlled phase shifter that is modified from a frequency-discriminator. The performance of this delay-time-tunable, modelocked EDFL including its properties of pulsewidth, tuning range, responsivity, and linearity will also be discussed.

The key element of this delay-time-tunable EDFL is a voltage-controlled and frequencydiscriminated phase shifter, which is constructed by using a voltage-controlled oscillator (VCO), an integral feedback (loop filter) circuit, a radio-wave frequency synthesizer (RFS), two frequency dividers (or so-called prescalars), a phase frequency detector (PFD), a temperature-controlled voltage source $\left(V_{\mathrm{REF}}\right)$, and a second-order active loop filter (LF). Several frequency prescaler ICs (MC120XX series) with divisors of 2,10 , and 256 are used to build up the frequency dividing modules with total devisor of 5120 for the VCO and RFS. The frequency-divided signals from the VCO and the RFS are phase-compared each other to generate an error voltage corresponding to the frequency/phase deviation between two signals. In general, there are two different types of PFDs that can be employed: one is the analog multiplier based PFD ICs (such as EXAR XR2208 or Harris ICL8013), and the other is the digital PFD ICs (such as Motorola MC4044 or 12040 series). The analog multiplier ICs although exhibit advantage of high sensitivity, but suffers from more drawbacks such as low phase-detection range $\left(<180^{\circ}\right)$ and inputlevel dependent gain constant. In contrary, the maximum phase-detecting range of a digital PFD can be up to $4 \pi$, which is particularly suitable for our proposed PLL-PS circuitry due to its linear transfer function within such a large phase-tuning range. In addition, the gain coefficient of the digital PFD is constant. The temperature-controlled voltage source is constructed by use of a temperature-stabilized and high precision voltage regulater (National Semiconductor, LM399) in connection with a resistor-based variable voltage tuning circuit. The second-order active LF is implemented by use of operational amplifier (Analog Device, OP27), RC, and discharging circuits. Experimentally, a microwave oscillator (VCO, HP8640B) operated in DC/FM mode is employed as the $\mathrm{VCO}$, which is frequency-discriminated to a frequency synthesizer (RFS, HP8648A) operated at a harmonic frequency of the linear repetition rate. The frequency of the VCO is synchronized to the RFS and the error voltage feedback to the VCO exhibits only a DC component. The timing jitter of the EDFL can thus be suppressed under the frequency discriminating condition. However, the error voltage can be further added with a controlling voltage $\left(V_{\mathrm{REF}}\right)$ to achieve the adjustment of the relative phase shift as well as the delaytime between VCO and RFS $[18,19]$. This leads to a voltage-controlled phase difference between the signals from RFS and locked VCO. Moreover, there are two different operating modes designed for the proposed laser. In free-running mode, the reference clock is coming from the RFS as aforementioned. However, the reference clock may also come from the signal optoelectronic converted from output of the EDFL for the laser operated at a feedback controlling mode (as shown by a dotted line in Fig. 1). In this case, mode-locking of EDFL can directly be built up by synchronizing the phase of modes involved in the amplified spontaneous emission (ASE) spectrum.

In more details, the basic principle of the frequency-discriminated phase shifter can be explained by deducing the phase change of the VCO from the equivalent noise model of the the circuitry schematically shown in Fig. 2. By using the nodal analysis, we can express the effect of the additional controlling voltage $\left(V_{\mathrm{REF}}\right)$ on the phase shift of the output signal from VCO as: 


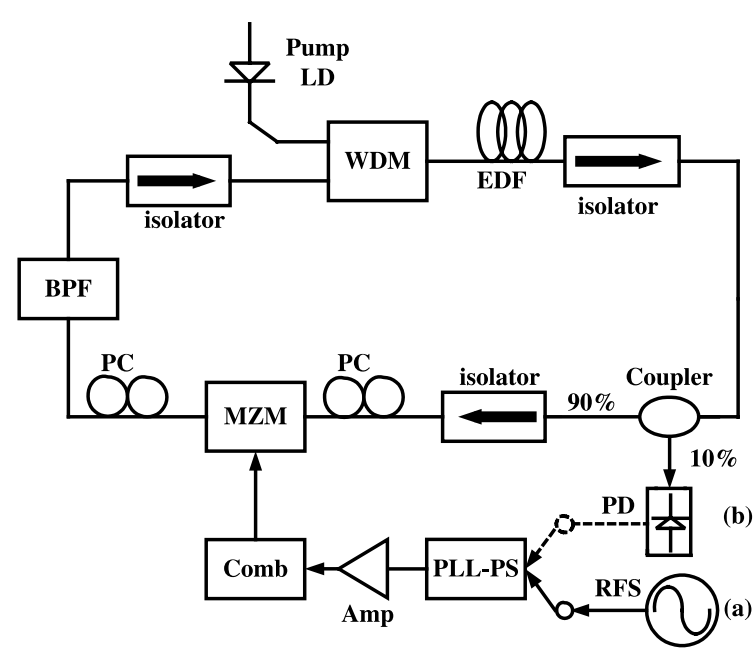

Fig. 1. The setup of a delay-time-tunable, mode-locked erbiumdoped fiber ring laser with (a) free-running mode (reference clock coming from RFS) and (b) feedback controlling mode (reference clock coming from PD). WDM: wavelength-division multiplexing coupler; EDF: erbium-doped fiber; PC: polarization controller; MZM: Mach-Zehnder intensity modulator; PD: high-speed photodetector; PLL-PS: phase-locked-loop phase shifter; DSO: digital sampling oscillator; Comb: comb generator (optional); BPF: band-pass filter.

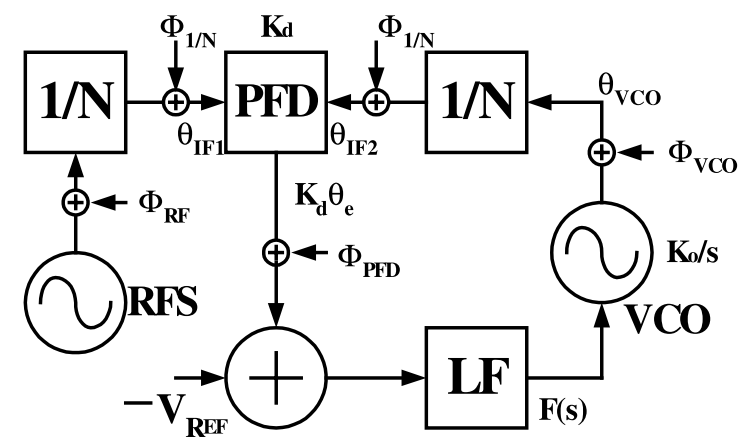

Fig. 2. The configuration and equivalent noise model of the frequency-discriminated phase shifter. RFS: radio-wave frequency synthesizer; LF: loop filter; PFD: phase frequency detector; VCO: voltage controlled oscillator; $1 / N$ : the frequency divider.

$$
\begin{aligned}
& \theta_{\mathrm{e}}=\theta_{\mathrm{IF} 1}-\theta_{\mathrm{IF} 2} \\
& \theta_{\mathrm{IF} 1}=\left[\frac{\Phi_{\mathrm{RFS}}}{N}+\Phi_{1 / N}\right] ; \quad \theta_{\mathrm{IF} 2}=\left[\frac{\Phi_{\mathrm{VCO}}}{N}+\Phi_{1 / N}\right]+\theta_{\mathrm{VCO}} \\
& \theta_{\mathrm{VCO}}=\left[K_{\mathrm{d}} \theta_{e}+N_{S}\left(\Phi_{\mathrm{PFD}}\right)-V_{\mathrm{REF}}\right] F(s) K_{0} / s+\Phi_{\mathrm{VCO}}
\end{aligned}
$$

where $\theta_{\mathrm{e}}$ is the phase difference between the input signal and the reference at the phase detector, $\theta_{\mathrm{IF} 1}$ is the phase of the intermediate frequency (IF) reference signal which is frequency-divided from the RF synthesizer through the divider, $\theta_{\mathrm{IF} 2}$ is the phase of the intermediate frequency (IF) reference signal derived from the VCO through another frequency divider, and $\theta_{\mathrm{VCO}}$ is the phase of the VCO under closed-loop condition. The single-sideband (SSB) phase noise of each component is denoted by $\Phi$ with corresponding sub-indices. For example, $\Phi_{\mathrm{RFS}}, \Phi_{1 / N}, \Phi_{\mathrm{PFD}}$, and $\Phi_{\mathrm{VCO}}$ denote the corresponding SSB phase noises of the reference frequency synthesizer, frequency divider, phase/ frequency detector, and voltage controlled coscillator, respectively. In addition, $F(s)$ is the Laplacetransformed transfer function of the second-order active loop filter (LF), the $K_{\mathrm{d}}$ and $K_{0}$ are the gain constants of the phase detector and the VCO respectively. In Eq. (1), we have assumed that an offset voltage $\left(V_{\mathrm{REF}}\right)$ is added prior to the LF. By substituting $\theta_{\mathrm{IF} 1}$ and $\theta_{\mathrm{IF} 2}$ into $\theta_{\mathrm{e}}$, we can rewrite the phase change of the output signal from the VCO which is phase-locked to the clock signal:

$$
\begin{aligned}
\theta_{\mathrm{VCO}} \approx & \frac{\left\{K_{\mathrm{d}}\left(\frac{\Phi_{\mathrm{RF}}-\Phi_{\mathrm{VCO}}}{N}\right)+N_{\mathrm{s}}\left(\Phi_{\mathrm{PD}}\right)-V_{\mathrm{REF}}\right\} F(s) K_{0} / s}{1+K_{\mathrm{d}} F(s) K_{0} / s} \\
& +\frac{\Phi_{\mathrm{VCO}}}{1+K_{\mathrm{d}} F(s) K_{0} / s} \\
\approx & \Phi_{\mathrm{PLL}}-V_{\mathrm{REF}} \frac{F(s) K_{0} / s}{1+K_{\mathrm{d}} F(s) K_{o} / s} \\
\equiv & \Phi_{\mathrm{PLL}}+\Delta \theta_{\mathrm{VCO}} .
\end{aligned}
$$

Here $\Delta \theta_{\mathrm{VCO}}$ is the phase shift of the VCO caused by adding $V_{\text {REF }}$. Eq. (2) therefore represents the effect of the offset voltage $V_{\mathrm{REF}}$ on the phase change of the microwave signal from the VCO. Owing to a relatively large loop gain in the passband, we can make the following approximation:

$\frac{F(s) K_{0} / s}{1+K_{\mathrm{d}} F(s) K_{0} / s} \approx 1 / K_{\mathrm{d}}$.

As a result, the phase change $\Delta \theta_{\mathrm{VCO}}$ of the microwave signal from the frequency-discriminated VCO is approximately in linear proportion to the offset voltage $\left(V_{\mathrm{REF}}\right)$ as follows: 


$$
\begin{aligned}
\Delta \theta_{\mathrm{VCO}} & =V_{\mathrm{REF}} \frac{F(s) K_{0} / s}{1+K_{\mathrm{d}} F(s) K_{0} / s} \approx V_{\mathrm{REF}} / K_{\mathrm{d}} \\
& \equiv R_{\mathrm{d}} V_{\mathrm{REF}} .
\end{aligned}
$$

That is, the phase change of the microwave signal as well as the delay-time of the EDFL controlled by the VCO based servo-loop can be linearly changed by tuning the DC voltage $\left(V_{\mathrm{REF}}\right)$ without the use of high- frequency circuit components. The parameter $R_{\mathrm{d}} \approx 1 / K_{\mathrm{d}}$ (with a unit of degree/volt) represents the phase-tuning sensitivity. Choosing a phase comparator with a larger phase-gain constant $K_{\mathrm{d}}$ is mandatory, however, will also lead to smaller phase sensitivity and a reduced phase-shifting range. Therefore, an optimal design of the phase-comparing circuit for achieving larger phase sensitivity without degrading the phase-comparing ability is needed for practical applications.

Fig. 2 shows the block diagram of actively mode-locked EDFL operated in either free-running mode or delay-time controlled mode. The EDFL consists of a unidirectional ring cavity, which is constructed by using a EDF with length of $3 \mathrm{~m}$ (as the gain medium), a laser diode at 978 $\mathrm{nm}$ (as the pumping source), a $1 \times 2$ coupler with $90 / 10$ output coupling ratio, three fiber-based polarization-independent optical isolators (although one should be enough), two polarization controllers, an optical band-pass filter with $3 \mathrm{~dB}$ bandwidth of $0.7 \mathrm{~nm}$ (wavelength tunable), and a Mach-Zehnder intensity modulator with a $3 \mathrm{~dB}$ bandwidth greater than $10 \mathrm{GHz}$ (acts as a active mode-locker). The total length of the EDF ring laser cavity is $16 \mathrm{~m}$, which corresponds to a fundamental cavity mode spacing of $12.5101 \mathrm{MHz}$. The mode-locked condition is achieved by tuning the frequency of the RFS $\left(f_{\mathrm{h}}\right)$ to match a higher order harmonic component (with an integral multiple $N)$ of the cavity mode $\left(f_{\mathrm{h}}=500.404\right.$ $\mathrm{MHz}, N=40$ ). The VCO signal is further amplified by a microwave power amplifier with a gain of $27 \mathrm{~dB}$, and then sent into a comb generator to output an electrical pulse train for driving the active mode-locker. This facilitates a more stable mode-locking performance with reduced timing jitter. The optical pulse-train generated from the actively mode-locked EDFL is monitored by using a high-speed photodetector $\left(f_{3 \mathrm{~dB}}=45 \mathrm{GHz}\right)$ and a sampling oscilloscope $\left(f_{3 \mathrm{~dB}}=50 \mathrm{GHz}\right)$.

The temporal trace of the optical pulse-train generated from the mode-locked EDFL under the control of $V_{\mathrm{REF}}$ is simultaneously monitored by DSO. The leading and delayed optical pulse-trains with respect to the original one are detected and shown in Figs. 5(a) and (b). The upper trace in each figure illustrates the original $500.404 \mathrm{MHz}$ optical pulse-train from the mode-locked fiber ring laser without tuning the $V_{\mathrm{REF}}$ of frequency-discriminated phase shifter. As $V_{\mathrm{REF}}$ tuned from 0 to $-3.35 \mathrm{~V}$, the pulse train is found to be delayed by 0.9 ns (see in Fig. 3(a)). Alternately, a leading time of $1 \mathrm{~ns}$ for the pulse train by setting $V_{\mathrm{REF}}=3.6 \mathrm{~V}$ can be seen in Fig. 3(b). By changing the $V_{\text {REF }}$ from -3.35 to $3.6 \mathrm{~V}$, the laser pulse-train is found to shift from -0.9 to $1 \mathrm{~ns}$ with respect to the RFS signal as well as the originally un-shifted optical pulse-train. This tuning range of the delay-time thus corresponds to nearly one period of the laser
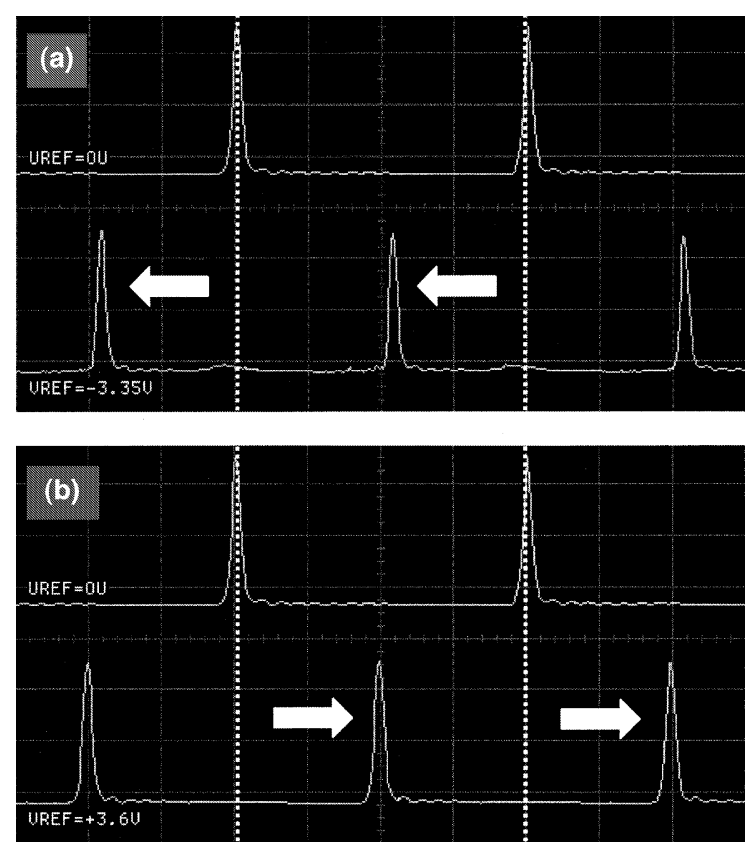

Fig. 3. The time-delayed optical pulse-train repeated at 500.404 $\mathrm{MHz}$ controlled at different $V_{\mathrm{REF}}$ with respect to the original one (upper trace). The lower traces in (a) and (b) show the optical pulse-train delayed by setting $V_{\mathrm{REF}}$ as -3.35 and $3.6 \mathrm{~V}$, respectively ( $x$-axis: temporal span in $500 \mathrm{ps} / \mathrm{div}$ ). 
pulse train, i.e., a maximum phase-tuning range close to $2 \pi$. Note that the optical pulsewidth of the mode-locked EDF ring laser maintains constant ( $\tau_{\text {FWHM }} \approx 59$ ps with the adding band-pass filter) during the delay-time tuning procedure. The relative phase shift as well as the delay-time of the optical pulse-train as a function of $V_{\mathrm{REF}}$ is shown in Fig. 4. The maximum phase-shifting range of the laser pulse train is up to $1.9 \pi$, which corresponds to a maximum temporal duration of about 0.95 period (also corresponding to a delay-time of $\sim 1.9 \mathrm{~ns}$ at a repetition rate of $500.404 \mathrm{MHz}$ ). It is worthy mentioning that neither the pulse repetition rate nor the optical pulsewidth is changed during the delay-time tuning process since the frequency-discriminated phase shifter is working at synchronous condition. The tuning linearity of the frequency-discriminated phase shifter as a function of controlling voltage $\left(V_{\mathrm{REF}}\right)$ is nearly sinusoidal with a period of of $3.2 \mathrm{~V}$ and the estimated maximum deviation $(\Delta \theta / \theta)$ is $< \pm 5 \%$ (see Fig. 5). The tuning responsivity of the EDFL pulse-train is calculated to be $0.27 \mathrm{ps} / \mathrm{mV}$, which corresponds to a tuning resolution of $0.5 \mathrm{ps}$, limited by the voltage fluctuations of about $2 \mathrm{mV}$ in our current system. It is estimated that a relative delay-time of $10 \mathrm{ps}$ is already enough for establishing an OTDM system with the bit-rate up to $100 \mathrm{~Gb} / \mathrm{s}$ by using an array of our proposed delaytime-tunable mode-locked EDFLs. On the other

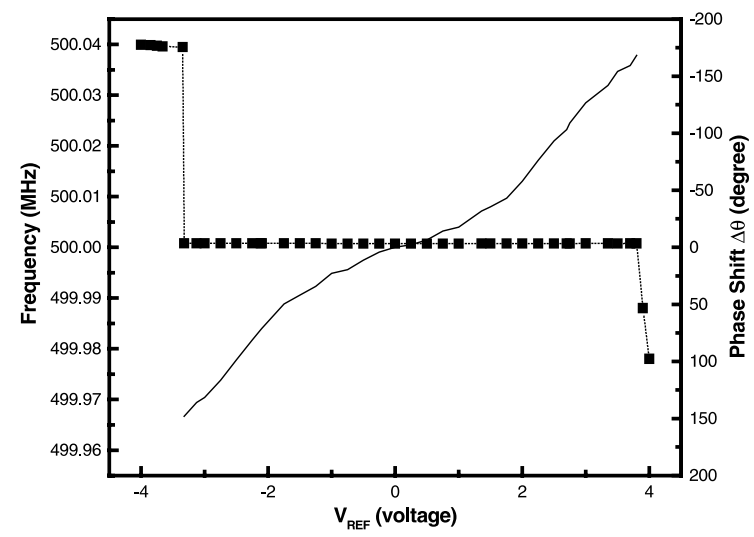

Fig. 4. The repetition rate (solid square with dashed line) and the relative delay-time (solid line) of the optical pulse-train from mode-locked EDFL plotted as a function of controlling voltage $\left(V_{\mathrm{REF}}\right)$.

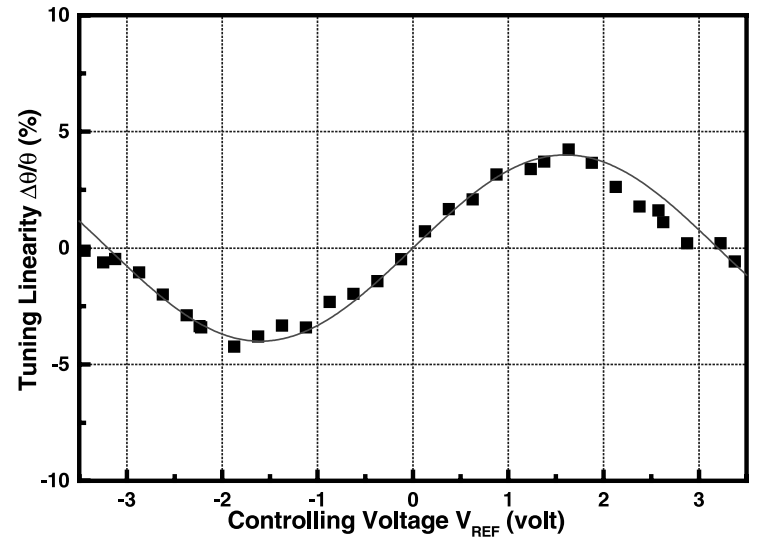

Fig. 5. The tuning linearity as a function of controlling voltage $\left(V_{\mathrm{REF}}\right)$ is nearly sinusoidal with period and maximum estimated deviation $(\Delta \theta / \theta)$.

hand, enhancing the tuning resolution of the current system is still mandatory for high-speed optical sampling measurements. In comparison with commercially available analog microwave phase shifters, the demonstrated frequency-discriminated phase shifter has been shown to exhibit better performances on pulse-tuning linearity and maximum shifting range. Nonetheless, further improvement on the tuning linearity and the shifting range of the system to reach the theoretical limits are straightforward and should be achievable via the use of a PFD with higher operating bandwidth and shorter rising/falling time.

Finally, the single-sided-band (SSB) phase noise spectra of the delay-time-tunable mode-locked EDFL operated at free running (dotted line) and feedback controlling (solid line) modes are measured, respectively. The single-side-band (SSB) phase noise spectrum is measured by using a highspeed photodetector (New Focus 1014, $f_{3 \mathrm{~dB}}>45$ $\mathrm{GHz}$ ) in connection with a DC-signal blocker and a microwave spectrum analyzer (Rhode and Schwartz FSEK30, $f_{3 \mathrm{~dB}}=40 \mathrm{GHz}$ ) with resolution bandwidth of $1 \mathrm{~Hz}$. The SSB phase noise density spectrum is taken from the double-side-band spectrum associated with its power level of the central peak normalizing to $0 \mathrm{dBm}$. The Rhode and Schwartz offers a standard programmable for extraction of the SSB phase noise density spectrum from the general spectrum. The carrier frequency (for 
example, $f_{\text {carrier }}=500.404 \mathrm{MHz}$ ) is offset to zero and positioned at left margin of the figure when transferring the SSB phase noise spectrum. The scanning time is set as $60 \mathrm{~s}$. The resolution bandwidth is $1 \mathrm{~Hz}$ at offset frequency of lower than 200 $\mathrm{Hz}$, which is subsequently increasing up to 5 and 10 $\mathrm{Hz}$ as the offset frequency increases up to 1 and 5 $\mathrm{kHz}$, respectively. Note that the system limitation of about $-120 \mathrm{dBc} / \mathrm{Hz}$. In Fig. 6 , it is observed that the instantaneous SSB phase noise of the EDFL operated at free-running mode is higher than -50 $\mathrm{dBc} / \mathrm{Hz}$ (measured at offset frequency of less than 50 $\mathrm{Hz}$ ), which further decreases down to $-70 \mathrm{dBc} / \mathrm{Hz}$ at offset frequency of $100 \mathrm{~Hz}$ or larger. When operating at feedback controlling mode, the SSB phase noise of the EDFL is significantly reduced by $10 \mathrm{dBc} / \mathrm{Hz}$ or larger at offset frequencies of $>100 \mathrm{~Hz}$ as compared to that of the laser operated at freerunning mode. In general, the SSB phase noise of the optical pulse generated from the feedback controlled EDFL can remain as $<-105 \mathrm{dBc} / \mathrm{Hz}$ at offset frequency of $1 \mathrm{kHz}$ or larger, which is only $10 \mathrm{~dB}$ larger than that of the RFS used in our experiment. It is observed that the noise density spectrum at offset frequencies below $100 \mathrm{~Hz}$ is greatly fluctuated due to the frequency independence between the RFS and EDFL longitudinal mode at free-running mode. This indicates there are still a residual frequency drift (or modulation) of the longitudinal mode of the EDFL cavity with a free-running mode-locker due to thermal and environmental

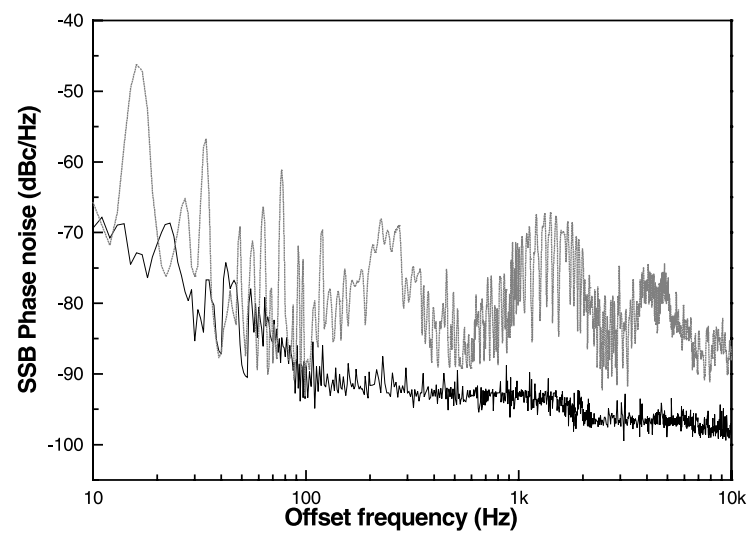

Fig. 6. The single-sided-band phase noise spectra of the delaytime-tunable mode-locked EDFL operated at free running (dotted line) and feedback controlling (solid line) modes. fluctuations, which significantly changes the noise spectrum in low offset frequency region $\left(f_{\text {offset }}<100\right.$ $\mathrm{Hz}$ ) at different scans (with scanning period of 5-60 s under a frequency span of $10 \mathrm{kHz}$ ). This inevitably causes the unexpected spectrum dip at lower offset frequency region during scans. Indeed, the excellent tracking performance of the PLL-PS facilitates the noise suppression of the mode-locked EDFL at offset frequencies within the pass-band of the loop filter in the PLL-PS. The further reduction in phase noise density at low offset frequencies $\left(f_{\text {offset }}<100\right.$ $\mathrm{Hz}$ ) depends strictly on both the noise figure of the electrical frequency reference (under the frequencytracking mode) the stability control of the EDFL cavity itself (under the regeneratively mode-locking mode). Nonetheless, the feedback controlling and fast tracking capability of the PLL-PS has efficiently reduced the noise densities. These results interpret that the feedback controlled operation would be promising for certain applications requiring optical pulses with low phase noises and jitters.

In conclusion, we have demonstrated a jittersuppressed and delay-time-tunable mode-locked EDFL by using a modified frequency-discriminated phase shifting circuit as the pulse delay-time controller. The optical pulse-train can be synchronized and phase-tuned with respect to the external microwave clock. By changing the voltage from -3.35 to $3.6 \mathrm{~V}$, the maximum tuning range of the phase and the delay-time of the $500.404 \mathrm{MHz}$ optical pulse-train from the laser can be up to $340^{\circ}$ $(\sim 1.9 \pi)$ and 1.9 ns (nearly 1 period) respectively under a tuning responsivity of $0.27 \mathrm{ps} / \mathrm{mV}$. The tuning linearity as a function of the controlling voltage is slightly sinusoidal with maximum estimated deviation is $< \pm 5 \%$. The SSB phase noise of the laser operated at feedback-controlled mode is significantly reduced to the system limitation of about $-120 \mathrm{dBc} / \mathrm{Hz}$ (measured at offset frequency of $100 \mathrm{kH}$ ), which is at least $7 \mathrm{~dB}$ smaller than that of the laser operated at free-running mode. Highspeed delay-time keying is possible via programmable control of the $V_{\mathrm{REF}}$ in the servo-loop. Extension of this technique to a higher repetition rate should be possible and it is expected that such lasers should find many important applications in high bit-rate OTDM and EOS systems. 


\section{Acknowledgements}

This work was supported in part by the National Science Council (NSC) of the Republic of China under Grants NSC89-2215-E-027-007 and NSC90-2215-E-027-008. The authors appreciate Mr. Y.-L. Cheng for his support in taking some of the experimental data.

\section{References}

[1] H. Takara, S. Kawanishi, M. Saruwatari, K. Noguchi, Electron Lett. 28 (1992) 2095.

[2] T. Pfeiffer, G. Veith, Electron Lett. 29 (1993) 1849.

[3] K.K. Gupta, D. Novak, Electron Lett. 23 (1997) 1330.

[4] S. Kawanishi, K. Okamoto, M. Ishii, O. Kamatani, H. Takara, K. Uchiyama, Electron Lett. 33 (1997) 976.

[5] N. de Baynes, J. Allan, J.R.A. Cleaver, IEEE Microwave Guided Wave Lett. 6 (1996) 126.

[6] E. Yoshida, M. Nakazawa, Electron Lett. 34 (1998) 1753.

[7] M. Nakazawa, E. Yoshida, Electron Lett. 32 (1996) 1291.
[8] M. Nakazawa, E. Yoshida, H. Kubota, Y. Kimura, Electron Lett. 30 (1994) 2038.

[9] A.D. Ellis, R.J. Manning, I.D. Phillips, D. Nesset, Electron Lett. 35 (1999) 645.

[10] M. Nakazawa, K. Tamura, E. Yoshida, Electron Lett. 32 (1996) 461.

[11] B. Bakhshi, P.A. Andrekson, X. Zhang, Opt. Fiber Tech. 4 (1998) 293.

[12] R. Taylor, S. Forrest, IEEE Photon. Tech. Lett. 10 (1998) 144.

[13] M.Y. Frankel, R.D. Esman, IEEE Trans. Microwave Theory and Tech. 43 (1992) 2387.

[14] J. Stulemeijer, F.E. van Vliet, K.W. Benoist, D.H.P. Maat, M.K. Smit, IEEE Photon. Tech. Lett. 11 (1999) 122.

[15] J.B. Georges, R.A. Lux, S.P. Yeung, K.Y. Lau, W. Chang, IEEE. Photon. Tech. Lett. 8 (1996) 953.

[16] J.L. Corral, J. Marti, J.M. Fuster, IEEE Trans. Microwave Theory and Tech. 47 (1999) 1315.

[17] K. Ghorbani, A. Mitchell, R.B. Waterhouse, M.W. Austin, IEEE Trans. Microwave Theory and Tech. 47 (1999) 645.

[18] G.-R. Lin, T.-S. Hwang, Y.-H. Chuang, S.-C. Wang, C.-L. Pan, IEEE Trans. Microwave Theory and Tech. 46 (1998) 1419.

[19] G.-R. Lin, Opt. Lett. 25 (2000) 799. 